\title{
Electronic states on the surface of graphite
}

\author{
Guohong Li, Adina Luican, Eva Y. Andrei*
}

Department of Physics and Astronomy, Rutgers Univsersity, Piscataway, NJ 08854, USA

Elsevier use only: Received date here; revised date here; accepted date here

\begin{abstract}
Graphite consists of graphene layers in an AB (Bernal) stacking arrangement. The introduction of defects can reduce the coupling between the top graphene layers and the bulk crystal producing new electronic states that reflect the degree of coupling. We employ low temperature high magnetic field scanning tunneling microscopy (STM) and spectroscopy (STS) to access these states and study their evolution with the degree of coupling. STS in magnetic field directly probes the dimensionality of electronic states. Thus two dimensional states produce a discrete series of Landau levels while three dimensional states form Landau bands providing a clear distinction between completely decoupled top layers and ones that are coupled to the substrate. We show that the completely decoupled layers are characterized by a single sequence of Landau levels with square root dependence on field and level index indicative of massless Dirac fermions. In contrast weakly coupled bilayers produce special sequences reflecting the degree of coupling, and multilayers produce sequences reflecting the coexistence of massless and massive Dirac fermions. In addition we show that the graphite surface is soft and that an STM tip can be quite invasive when brought too close to the surface and that there is a characteristic tip-sample distance beyond which the effect of sample tip interaction is negligible.
\end{abstract}

(C) 2009 Elsevier Science. All rights reserved

Keywords: A) surfaces and interfaces;(C) scanning tunneling microscopy; (D) quantum Hall effect;

* Corresponding author. Tel.: +1-732-445-2509; fax: +1-732-445-4343; e-mail: eandrei@phyiscs.rutgers.edu 


\section{Introduction}

Graphene, a one atom thick crystal of carbon arranged in a two-dimensional (2d) honeycomb lattice, posses extraordinary electronic properties. Its charge carriers are quasiparticle whose motion, being governed by the Dirac-Weyl equation, mimics that of quantum relativistic particles (massless Dirac fermions) in (2+1) dimensions[1-6]. The energymomentum dispersion is conical with a slope corresponding to a quasiparticle velocity $\mathrm{v}_{\mathrm{F}} \sim \mathrm{c} / 300$ (c is the speed of light) and an apex, the Dirac point, that separates between particles (electrons) and antiparticles (holes). At charge neutrality the Fermi energy, $E_{F}$, coincides with the Dirac point. In the presence of a perpendicular magnetic field $B$, the spectrum becomes a discrete series of Landau levels forming an unusual sequence [4-6]

$E_{n}=\operatorname{sgn}(n)\left(2 e \hbar v_{F}|n| B\right)^{1 / 2}$;

where $\hbar$ is Planck's constant over $2 \pi$, $-e$ is the electron charge, $n=\ldots-2,-1,0,1,2 \ldots$ with $n>0$ corresponding to electrons and $n<0$ to holes. The unusual zero energy level at $n=0$ is a consequence of the chirality of the Dirac fermions and consists of an equal number of electron and hole states. This Landau level leads to the absence of a Hall plateau at zero filling factor and to the $1 / 2$ shift in the plateau sequence compared to that seen in standard 2d electron systems obeying the Schroedinger equation [3-8].

When two graphene layers are stacked forming a bilayer, the interlayer coupling leads to the appearance of mass. However, the resulting massive fermion quasiparticles are still chiral, with a Landau level at zero energy, and an unusual level sequence $[9,10]$

$E_{n}=\operatorname{sgn}(n) \hbar \omega_{c}\left(|n|(|n|-1)^{1 / 2}\right.$

where $\omega_{c}=e B / m *$ with $m *$ the effective mass. It can be seen that $\mathrm{E}_{0}=\mathrm{E}_{ \pm 1}=0$.

As the number of layers is increased, massive and massive Landau level sequences are expected to coexist [11] and eventually develop into the Landau band of bulk graphite [12].

Novel quantum Hall effect sequences, attributed to the unusual Landau levels, were observed by transport measurements on graphene and bilayer graphite films extracted from bulk graphite [7-9]. However, more advanced techniques are needed to observe Landau levels directly. Scanning tunneling microscope (STM) is a powerful tool to measure the Landau levels because one can bias the sample voltage to select specific energy levels for electrons to tunnel between the scanning tip and the sample. Sweeping the sample bias in a scanning tunneling spectroscopy experiment reveals the density of states of electrons in the sample with each Landau level producing a peak in the tunneling spectrum.

Graphite, particularly highly oriented pyrolytic graphite (HOPG), is a common substrate for STM studies. It is well known that various defects exist on the surface of HOPG after cleavage $[13,14]$. Here we show that the defects could lead to decoupling of the top graphene layer from the bulk graphite affecting the electronic states on the surface significantly. As we show below this makes it possible to access novel electronic states of the graphite surface with various degrees of coupling to the bulk. In the limit of complete decoupling we find that the top layer exhibits the structural and spectroscopic properties of a single graphene layer.

\section{Experiments}

The experiments were conducted with a home built, low temperature high magnetic field scanning tunneling microscope [15]. The STM tips were obtained from mechanically cut Pt-Ir wire and the samples were HOPG cleaved in air. We measured tunneling conductance $\mathrm{dI} / \mathrm{dV}$ using lock-in detection with ac modulation of the sample bias voltage at 450 $\mathrm{Hz}$ or $340 \mathrm{~Hz}$. Magnetic fields were applied normal to sample surface. All measurements were carried out at $4.4 \mathrm{~K}$. Cooling to liquid helium temperatures was mediated by helium exchange gas $(0.65$ Torr at room temperature).

\section{Tip-sample interaction}

Scanning tunneling spectroscopy gives access to the local density of states [16]. However, as we show below, the STM tip is not necessarily noninvasive for 
a soft sample like graphite [17]. Fig.1 illustrates the evolution of tunneling spectra with tip-sample distance, which was controlled by varying the bias voltage for a given tunneling current, 50pA. The spectrum changed qualitatively for bias voltages below $20 \mathrm{mV}$. At high bias voltages, a Fermi level shift was observed though the spectra were qualitatively similar. For large tip-sample distances this shift of the Fermi level with bias voltage becomes negligible [15].

The tip-sample interaction is clearly seen in Fig.1 by comparing the two STM topography images which share all the same tunneling parameters except for the bias voltage settings that control the sampletip separation. A clear dent is seen to develop in the initially flat sample surface when the tip-sample separation becomes too small.

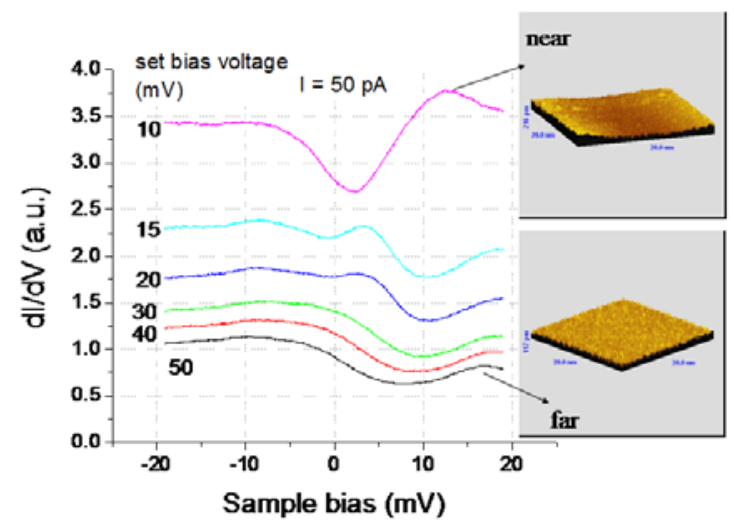

Fig. 1. Scanning tunneling spectroscopy for different tip-sample distance, which was controlled by the set bias voltage. Insets, STM images with size of $20 \mathrm{~nm}$ show a flat surface when the tip is far away from the sample surface but a dented one when the tip is near. Each spectrum was taken with ac modulation of $1 \mathrm{mV}_{\mathrm{rms}}$ and averaged over 256 spectra.

Another aspect of this anomaly is seen by plotting the tip position as a function of bias voltage for a series of tunneling currents shown in Fig.2(a). All curves exhibit a kink at a bias voltage below which a sudden increase in the slope suggests yielding of the sample surface. The onset of the low bias anomaly decreases with decreasing set tunneling current. Outside the anomaly regime tunneling currents depend exponentially on the tip-sample distance for a fixed bias voltage allowing to extract the apparent bias height [18], $96 \mathrm{meV}$ for the sample shown in Fig.2. For all the data showing tip-sample distance the origin is arbitrary.
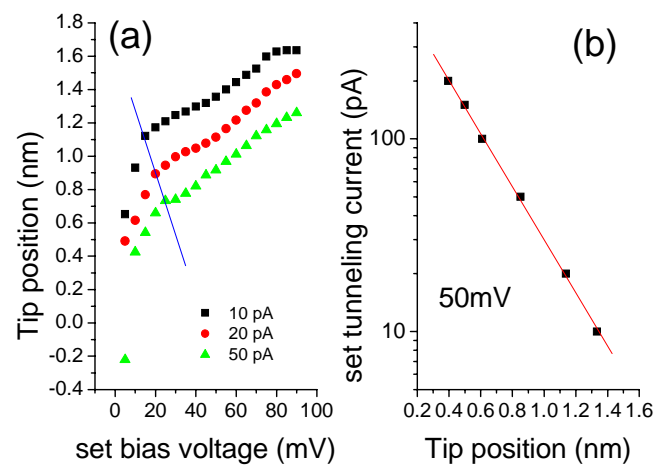

Fig. 2. (a) Tip position for different set tunneling currents for sample A. The line shows the onset of the low bias anomaly. (b) Tunneling current increases exponentially with decrease of tipsample distance for set bias voltage of $50 \mathrm{mV}$.

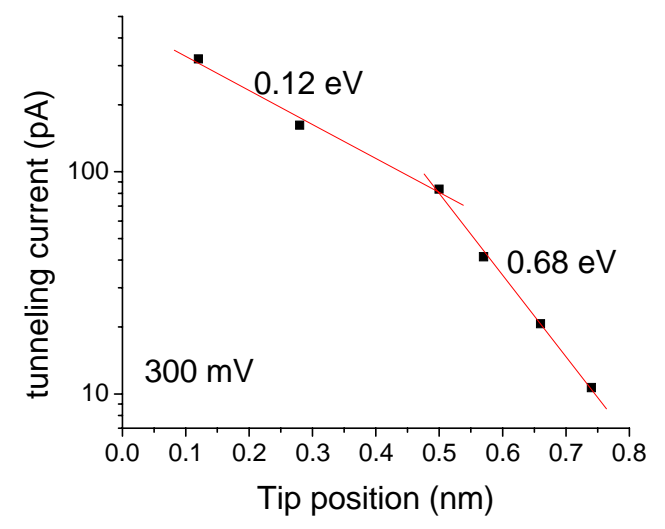

Fig. 3. Tunneling current versus tip position (with arbitrary reference) at set bias voltage of $300 \mathrm{mV}$ for sample $B$. Note the change of apparent barrier height from $0.68 \mathrm{eV}$ to $0.12 \mathrm{eV}$.

Fig.3 shows yet another sign of tip-sample interaction seen in a separate run with a sharper tip. When the tip was approached to the sample surface while keeping the bias voltage fixed at $300 \mathrm{mV}$, we found a decrease in the apparent barrier height from $0.68 \mathrm{eV}$ to $0.12 \mathrm{eV}$. At larger distances, for set currents below $80 \mathrm{pA}$, where the barrier was $0.68 \mathrm{eV}$ 
the normalized tunneling spectrum no longer changed with current. The $0.68 \mathrm{eV}$ barrier is comparable to the $0.6 \mathrm{eV}$ value obtained under moderate vacuum between a Pt surface and a $\mathrm{W}$ tip [18], however, the softening of the barrier was not observed for the hard surface of Pt [18].

These findings demonstrate that it is possible to attain reliable tunneling spectroscopy that reflects the intrinsic density of states on the surface of graphite by placing the STM tip at a height above which the spectrum becomes independent of tip-sample separation.

\section{Decoupled graphene layers}

The STM image of the freshly cleaved HOPG surface in Fig. 4 shows atomic steps associated with a graphene flake which is presumably lifted by hidden defects near the upper-left corner. In order to access the electronic states we measured scanning tunneling spectra at representative positions. It is useful to do such measurements in magnetic field so that decoupled graphene layers can be easily identified as discussed below. In a field of $4 \mathrm{~T}$, the region without flakes Fig. 4(b) shows a V-shaped spectrum without a clear peak structure, typical for a well-ordered graphite surface [19]. The spectra in, Figs. 4(a) and (d), exhibit anomalies associated with regions with structural defects in these regions. On the flake itself the spectrum, Fig. 4(c), exhibits a remarkably sharp and well defined sequence of peaks whose position varies with field. In contrast the spectra in Figs. 4(a), (b) and (d), which are almost independent of field.

The field dependence of the peaks in Fig. 4(c) is demonstrated in Fig.5(a) where the spectra in $2 \mathrm{~T}$ and 3T are compared. While most peaks fan out with increasing field, the center peak remains un-shifted, suggesting a reference point. Therefore we aligned the energy origin with this center peak Fig.5(b). In Fig.5(c) the major peaks aligned after scaling the $\mathrm{x}^{-}$ axis with $\mathrm{B}^{1 / 2}$.

The square-root scaling of the peak sequence with both field and level index, shown Fig.5(c), suggests that these are Landau level states resulting from $2 \mathrm{~d}$ massless Dirac fermions as described by Eq.(1). The only fitting parameter gives $\mathrm{v}_{\mathrm{F}}=1.0 \times 10^{6} \mathrm{~m} / \mathrm{s}$ for holes and $\mathrm{v}_{\mathrm{F}}=1.1 \times 10^{6} \mathrm{~m} / \mathrm{s}$ for electrons, which is consistent with results from transport measurements $[7,8]$.

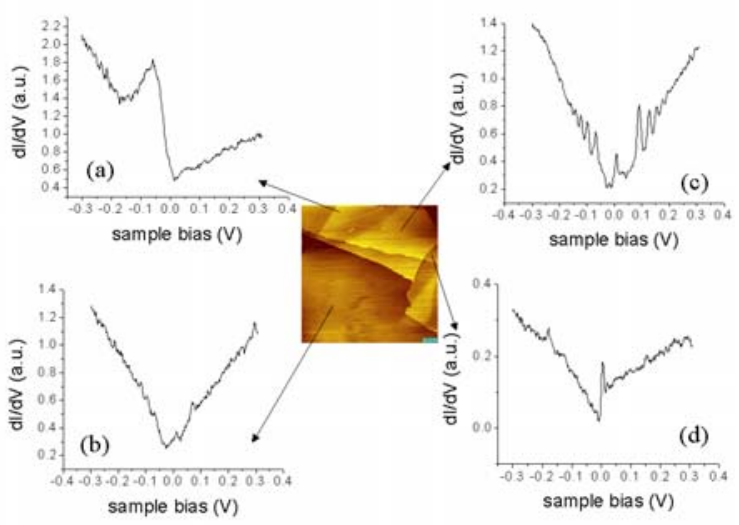

Fig. 4. (center) STM image (size: $1 \mu \mathrm{m} \times 1 \mu \mathrm{m}$, sample bias voltage $300 \mathrm{mV}$, tunneling current 20pA) of sample C. Arrows show typical areas where the scanning tunneling spectra (a) to (d) were taken. Magnetic field $4 \mathrm{~T}$.
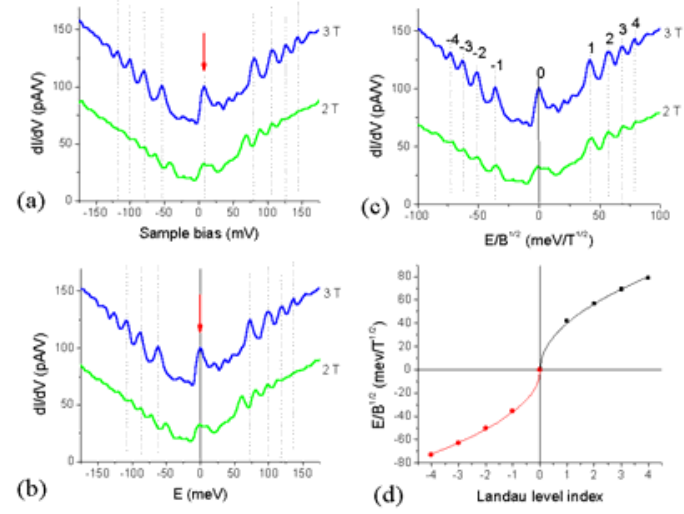

Fig. 5. (a) Field dependence of scanning tunneling spectroscopy for sample D (sample bias $300 \mathrm{mV}$, tunneling current 20pA). Arrow points to the peak whose position is independent of field, dashed lines highlight other major peaks in 3T spectrum. (b)Same as (a) with energy origin shifted so to the position of the peaks marked by arrows in (a). (c) Major peaks align after scaling the energy with $\mathrm{B}^{1 / 2}$. The Landau level index is marked by integers. (d) Square root dependence of scaled energy. Lines correspond to a fit to Eq. (1) with $\mathrm{v}_{\mathrm{F}}=1.0 \times 10^{6} \mathrm{~m} / \mathrm{s}$ for $\mathrm{n}<0$ and with $\mathrm{v}_{\mathrm{F}}=1.1 \times 10^{6} \mathrm{~m} / \mathrm{s}$ for $\mathrm{n}>0$.

The appearance of the Landau levels demonstrates that the top layers of cleaved graphite can decouple 
from the HOPG bulk. The degree of decoupling, which depends on the nature of the underlying surface and defects, is reflected in the Landau level sequence. For three or more (up to ten) coupled layers it was shown both theoretically [11] and experimentally [15] that the Landau level sequence separates into two coexisting series: One corresponding to massles Dirac fermions and follows the field and index dependence described by Eq.(1) and the other represents massive Dirac fermions described by Eq.(2). Two samples are compared in Fig.6. In both samples it is easy to recognize the presence of two Landau level sequences, corresponding to massless and to massive Dirac fermions. The massless sequences are similar but shifted with respect to the Femi level, indicating different doping levels. The massive sequence in sample A has a shorter period than that in sample B reflecting larger mass and stronger coupling to the substrate. We found that the coupling between the graphene layers is enhanced as the tip approached the sample surface [15].

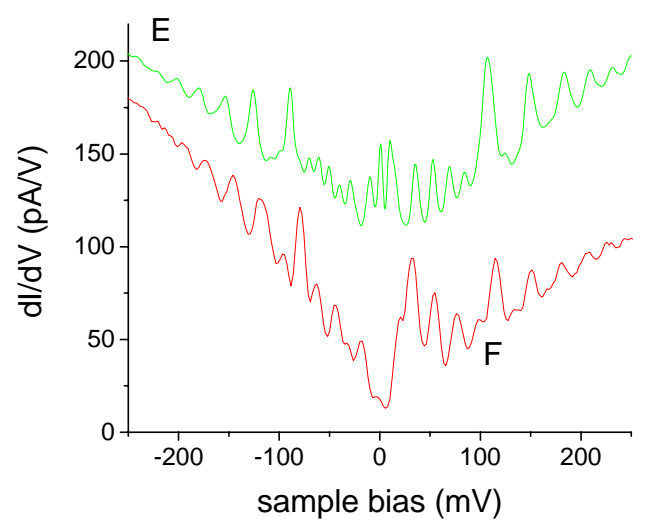

Fig. 6. Scanning tunneling spectroscopy of two samples in a field of $6 \mathrm{~T}$. The spectra were shifted vertically for clarity.

In the limit of vanishing interlayer coupling the electronic properties of the top layer becomes indistinguishable from that of single layer graphene, and the massive sequence near the Dirac point disappears [20], as shown in Fig.7(b). Here the Landau levels are so sharp that their widths are limited by electron-electron scattering [20,21]. It is interesting that the Fermi velocity $\mathrm{v}_{\mathrm{F}}$ in single layer graphene in Fig. 7 (b) is $\sim 20 \%$ smaller than that in multilayers [15]. The renormalization of $v_{\mathrm{F}}$ is attributed to electron-phonon interactions [15,20,22]. In addition we observe that the $n=0$ Landau level seen at the Dirac point splits into two levels separated by $\sim 10 \mathrm{meV}$. The origin of this splitting remains to be understood.

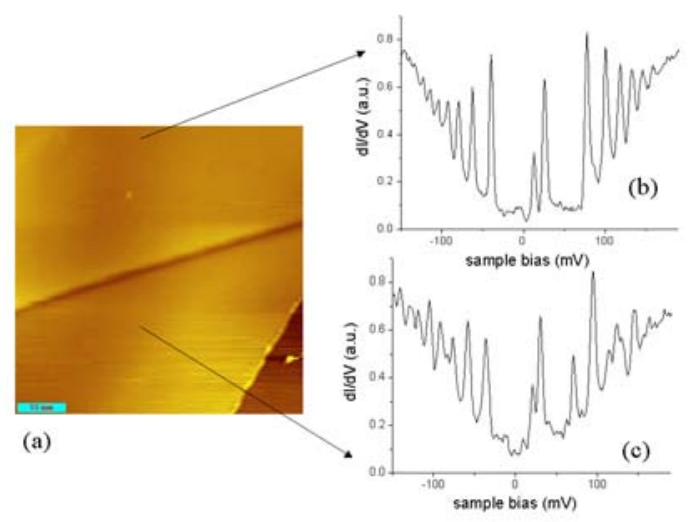

Fig. 7. (a) STM image of sample F (size: $300 \mathrm{~nm} \times 300 \mathrm{~nm}$, sample bias voltage $300 \mathrm{mV}$, tunneling current 20pA) Arrows show typical areas where the scanning tunneling spectra (b) and (c) were taken in a field of $4 \mathrm{~T}$.

Spectra taken in the lower part of Fig.7(c), which is separated from the decoupled layer by the ridge defect, show yet another sequence of Landau levels. They can neither be treated as a single sequence as in the single layer case, nor can they be described by coexisting massless and massive sequences as in the multi-layers. Further study [23] shows that these spectra represent Landau levels of a very weakly coupled graphene bilayer [24], with interlayer coupling strength of only $\sim 40 \mathrm{meV}$, one order of magnitude smaller than the interlayer coupling in bulk HOPG graphite.

\section{Summary}

This work presents a systematic STM study of graphene layers on graphite with special emphasis on exploring the effects of coupling to both the substrate and the tunneling tip. We have shown that an STM tip placed very close the graphite surface may significantly affect the tunneling spectra due to 
enhanced sample-tip interaction. At larger distances where the sample-tip interaction becomes negligibly small the tunneling spectra reflect the intrinsic density of states of the sample. In the presence of a strong magnetic field the tunneling spectra revealed Landau level sequences characteristic of the degree of coupling to the substrate. For completely decoupled single graphene layers we see a level sequence with square root dependence on both field and level index, providing a clear signature of massless Dirac fermion quasiparticles. In weakly coupled multilayers we find two coexisting sequences. One corresponding to maslless Dirac fermions and the other, which is linear in field and level index, corresponds to massive quasiparticles whose mass gives a direct measure of the degree of coupling.

Work supported by DE-FG02-99ER45742, NSFDMR-045673 and Lucent. We thank V. Kiryukhin for the HOPG crystal.

\section{References}

[1] P. R. Wallace, Phys. Rev. 71 (1947) 622.

[2] G. W. Semenoff, Phys. Rev. Lett. 53 (1984) 2449.

[3] F. D. M. Haldane, Phys. Rev. Lett. 61 (1988) 2015.

[4] Y. Zheng, T. Ando, Phys. Rev. B 65 (2002) 245420.

[5] V. P. Gusynin, S. G. Sharapov, Phys. Rev. Lett. 95 (2005) 146801.

[6] N. M. R. Peres, F. Guinea, A. H. Castro Neto Phys. Rev. B 73 (2006) 125411.

[7] K. S. Novoselov et al., Nature 438 (2005) 197.

[8] Y. Zhang et al., Nature 438 (2005) 201.

[9] K.S. Novoselov et al., Nature Phys. 2 (2006), 177.

[10] E. MaCann, V. I. Fal'ko, Y. Zhang, Phy. Rev. Lett. 96 (2006) 086805.

[11] F. Guinea, A. H. Castro Neto, N. M. R. Peres, Phys. Rev. B 73 (2006) 245426.

[12] G. Dresselhaus, Phys. Rev. B 10 (1974) 3602.

[13] C.R. Clemmer, T.P. Beebe Jr, Science 251 (1991) 640.

[14] H. Chang, A.J. Bard, Langmuir 7 (1991) 1143.

[15] G. Li, E.Y. Andrei, Nature Phys. 3 (2007) 623.

[16] J. Tersoff, D.R. Hamann, Phys. Rev. B 31 (1985) 805.

[17] J.M. Soler et al., Phys. Rev. Lett. 57 (1986) 444.

[18] G. Binnig et al. Appl. Phys. Lett. 40 (1982) 178.

[19] T. Matsui et al., Phys. Rev. Lett. 94 (2005) 226403.

[20] G. Li, A. Luican, E.Y. Andrei, arXiv.0803.4016.

[21] J. Gonalez et al., Phys. Rev. Lett. 77 (1996) 3589.

[22] C.-H. Park et al., Phys. Rev. Lett. 99 (2007) 086804.
[23] A. Luican, G. Li, E.Y. Andrei, Solid State Commun. (to appear).

[24] M. Pereira, F.M.Peeters, P. Vasilopoulos, Phys. Rev B 76 (2007) 115419. 